\title{
Variability of Loss Reserves
}

\author{
by Robert L. Brown
}




\title{
VARIABILITY OF LOSS RESERVES
}

\begin{abstract}
This paper addresses the issue of biases in the loss reserving process, some of which may be intentional. Using an empirical analysis of data from 169 companies over a seventeen year period, it is observed that the level of loss reserves exhibits cyclical behaviour, is different for companies of different sizes and is different for reinsurers than for direct insurers. Furthermore, after these factors are accounted for, the differences in levels between individual companies accounts for about three-quarters of the explainable variation.

The paper suggests that greater independence on the part of the loss reserve specialist could lead to more objective estimation and could reduce historical variability by about $40 \%$.
\end{abstract}




\section{Introduction}

Loss reserve estimates are made at least annually by one or more loss reserve specialists (now normally actuaries) in each insurance company writing property-casualty business. The degree of difficulty in estimating loss reserves depends on a number of factors. For example, some liability and other lines of business have long average time delays before settlement; the size of the ultimate loss may be positively correlated with the length of such delay; and the size of the ultimate loss may be highly variable.

The size of the loss reserve has an immediate impact on the income statement of the insurer since the year-to-year increase in loss reserves is a direct charge to incorne in the company's income statement. In theory, the size of loss reserves for a particular block of insurance business in successive years has no impact on the ultimate profitability of that block. The reserve only allocates portions of the profit to the successive years. In practice, however, the size of the loss reserves in successive years may influence the setting of premiums since incurred losses include estimates of loss reserves. Establishing inadequate reserves may lead to inadequate future premium income. Furthermore, a sharp increase in loss reserves has a direct influence on the income statement, shareholder confidence and stack prices.

Because the size of loss reserves affects income and hence taxes and stock prices, it is possible that the loss reserves in financial statements may not be objective estimates of future losses (see, for example, the article by Loomis, 1984). Grace (1990) hypothesizes that insurers are influenced by the desire to maximize earnings each year while maintaining a smooth progression of earnings in order to minimize investor uncertainty. This suggests that a company that is under-reserved in one year is likely to be under-reserved in the next year. In various empirical studies, Anderson (1973), Smith (1980) and Weiss (1985) all found that reserve errors had the effect of smoothing the underwriting income of insurers. This implies that the 'true' underwriting income stream is more volatile than that obtained using reserve estimates.

Because the insurance business is inherently risky and the estimation process is imperfect, 
variability in loss reserves is inevitable. The objective of this paper is to examine and test empirically various sources of variability, and test the hypothesis that reserve errors are nonrandom.

\section{Sources of Reserve Error}

The deviation of a reserve estimate from its 'true' value is termed the reserve error. The 'true' value of the reserve established at the end of an accounting year can only be established (or more accurately estimated) after all (or almost all) claims have been settled.

Some errors in reserving may be deliberate. Any attempt to smooth earnings is in this category. Forbes (1970), Smith (1980) and Weiss (1985) all confirmed that financial results for property-casualty companies were consistent with management's deliberate attempt to influence income smoothing through the reserving process. To the extent that a firm should be viewed from an on-going basis, this smoothing is considered a desirable characteristic of the loss reserving process by Pentikäinen and Rantala (1992). Of course, from a break-up perspective, such smoothing would be undesirable.

Deliberate over-reserving may be desirable to make the balance sheet of a company somewhat conservative. Reserves for property-casualty insurers have historically not included discounting of future cash flows even though reserves (or more correctly, assets of setting reserve liabilities) are invested. The classic argument is that such conservatism in reserving provides a margin of error against deviations in claims experience and against the effect of unanticipated inflation for claims that are not yet settled. This deliberate over-reserving is based on the belief that the balance sheet is more important than the income statement and that solvency considerations are paramount. The amount of any over-reserving plays the same role as surplus, but is hidden and thus protected from distribution to policy-owners or shareholders. Generally accepted accounting principles (GAAP) argue in favor of explicit recognition of the various sources of profit and a more 'accurate' income statement and hence, a more accurate balance sheet. Current tax regulation requires methods consistent 


\section{with GAAP.}

The Conference of Consulting Actuaries (1992) report that for the period 1987-1991, a total of 164 U.S. property-casualty insurance companies were declared insolvent and that the ultimate total surplus deficiency for these companies could reach $\$ 5$ billion. They report (p.91) that:

"The stated leading cause of insolvency is 'underreserving'. But in many cases, further analysis will show that this is a symptom rather than a cause. Underreserving can be a form of deferring the real problem. The practice of underreserving can lead to more easily defined causes.

When management recognizes there are serious problems, the easiest immediate solution is to seek justification for lower reserves. The loss reserve is an estimate of future costs for events that have occurred previously. Payments resulting from past events will be made over an extended period of time. The inherent delay in the loss reserve payout is often the basis for deliberate underreserving. In the case of deliberate underreserving, a further cause must usually be sought.

There may also be inadvertent underreserving, because future events may be hard to anticipate, or there may be a lack of understanding of the extent of loss. In instances where events giving rise to liabilities occur over an extended period of time, inadvertent underreserving can indeed be a cause of an insolvency."

As indicated by the last paragraph above, some sources of variation in loss reserves are non-deliberate. There are a variety of sources of such error. Some may be non-random and some may be random. Random errors occur when, for example, more claim-causing events ('accidents') than anticipated occur and when the sizes of losses associated with accidents are different from expected. This is often termed 'stochastic error'. 
Another source of error is 'model error'. Model error arises when the underlying mathematical model and associated method of estimation are inconsistent with reality. For example, many ratio-based methods, such as the chain ladder method (see, for example, van Eeghen, 1981) assume implicitly that the ratios of paid (or incurred) claims for successive years of development are constant. Estimation error is introduced to the extent that such ratios may vary from accident year to accident year. It is well-known that the chain ladder method under-estimates reserves when the outcomes are stochastic (Stanard, 1985).

Further error is introduced when a model is calibrated. This error is often called 'parameter error' since most models and hence methods are described in terms of parameters that require estimation based on a sample of previous years' data.

Pentikäinen and Rantala (1992) describe reserve volatility when the chain ladder method is used as well as when a premium-based loss reserving method is used. The premium basedmethod uses a percentage of the earned premium for each accident year in the calculation of reserves. To the extent that premiums are not good predictors of actual claims, model errors are introduced. Because it is well-known that an underwriting cycle exists, one would expect a similar cycle in loss reserves if they are based on premiums. Recently, Lamm-Tennant, Starks and Stokes (1992) analyzed loss ratios recognizing the nature of the cycle. They cite many references to the underwriting cycle. To the extent that there is systematic over- and under-pricing through the cycle, corresponding errors in loss reserves can occur when the methods used in loss reserving are linked to premium income rather than 'true' expected claims.

Stochastic error arises when 'the unexpected occurs'. Although many loss reserve methods are based on models that ignore stochastic variation in claims, there have been several methods that incorporate the stochastic component. Many are described in Taylor (1986) and the Institute of Actuaries' Claims Reserving Manual (1989). De Jong and Zehnwirth (1983) describe a general state-space model for loss reserving. This approach is used by Zehnwirth (1985). Verrall (1989a, 1989b, 1990) and Renshaw (1989) describe stochastic 
versions of the chain ladder method.

Modelling the stochastic error allows for the development of probability statements about the adequacy of a particular level of reserves. These probability statements can take into account both the stochastic error and the estimation error. However, they do not take any account of model error. The estimates are based on the assumption that the model (and hence the method) is appropriate.

Different methods yield different results because they are based on different model assumptions and/or different calibration methods (statistical estimation criteria). Methods are considered robust if they are rather insensitive to the model assumptions. The more robust a method is, the less sensitive it is to systematic variation that needs to be reflected in the reserve estimate. All reserving methods (and statistical estimation procedures) compromise robustness and sensitivity. Pentikäinen and Rantala (1992) try to address the issue of interpreting the different results from different methods, i.e. the model error.

\section{The Approach of this Paper}

Development of reserve estimates should involve selection of a model, calibration of the selected model and validation of the calibrated model. The estimate of loss reserves is a forecast. For models that include formal assumptions about the variability of the claims process (i.e. stochastic error), estimates of the likely variability of the forecast value can be obtained using statistical theory. In the practice of loss reserving, the forecasts are based on past payments or incurred claims, usually set up in the form of the standard 'runoff triangle'. This is done separately for each line of business. There is no standard way of combining the estimates of variability of various lines of business. If it is assumed that the experience of lines of business are stochastically independent, then the variance associated with a reserve estimate for all lines combined is the sum of the variances associated with each line. When the standard deviation is used as a measure of volatility, it is clear that variability decreases as lines of business are combined. However, if there is a strong positive correlation between 
lines, this does not hold.

The approach of this paper is to treat the whole company as the business entity, rather than treat each line of business separately. From the point of view of either the income statement (underwriting results) or the balance sheet (solvency) of an insurer, all lines of business are always combined. We will examine historical loss reserves and compare them with the corresponding subsequent runoff of claims. This will allow us to assess the performance of loss reserving in the past and to study the sources of variability in order to better explain the entire loss reserve process.

Since loss reserve specialists use different methods, estimates of variability for that specialist depend on the individual methods used. We will examine variability empirically by studying the variability for all companies combined, for each company over time, and for various subclasses of companies to provide a better explanation of the actual variability that should be anticipated regardless of the methods used.

We believe that it is inappropriate to impose solvency margins developed on a theoretical basis for any specific loss reserving method (e.g. chain ladder). Because of the different nature of claims information for different lines of business and for claims at different stages in the claim settlement process (e.g. incurred but not reported, reported but not yet settled), it is probably best to measure uncertainty of loss reserves on the basis of historical variability. In order to best understand historical variability, empirical studies are necessary.

Previous empirical studies of accuracy of loss reserves have been done by Forbes (1969, 1970), Anderson (1973), Ansley (1979) and Smith (1980), Aiuppa and Treischmann (1987), Grace (1990) and Panjer and Brown (1992) who each studied a collection of companies over a period of time. In particular, Grace examines the desire of companies to maximize firm value through the reserving process considering the tax status of the company. She also considers the desire of companies to smooth income by minimizing the variability of earnings. These are considered deliberate attempts to distort the true income picture of a company. This variation is specific to the individual company. 
There are sources of variability that are not specific to a single company. For example, the effect of inflationary increases in costs affects all insurers in a particular line of business such as automobile insurance. Such industry-wide influences should be observable as an annual effect across all companies in historical studies. Ansley (1979) studied the effect of inflation of reserve estimates.

Finally the level of conservatism inherent in reserves also varies from company to company. Small companies writing a few small lines of business have less ability to diversify variability than large companies writing more lines of business over which variability is not (or negatively) correlated. Hence, one would expect smaller companies to hold relatively larger reserves than large insurers.

Similarly, one might expect reinsurers that are part of world-wide reinsurance groups to hold relatively smaller reserves than small domestic insurers who do not have the ability to diversify risk internationally.

\section{The Data}

In this paper we try to identify the influences of various sources on the loss reserves by examining historical data from a set of 169 companies operating under federal regulation in Canada. This study is significantly larger than any of the cited previous studies, most of which examine only U.S. companies. The data were obtained from the annual statements over the period 1975-1991.

Companies are categorized by 'size' (Small, Medium and Large) on the basis of 1991 premium income, and by 'type' (Domestic or Foreign) on the basis of ownership. Reinsurers are separately identified.

For each year from 1975 to 1986 , the aggregate loss reserve for all lines of business (including loss adjustment expenses) for all prior accident years combined is compared with the runoff in the subsequent five years and any remaining loss reserve at that time. The difference is measured as a percentage excess or deficiency (see section 5 ). 
Using the runoff for five years for the most recent accident year means that, for the prior accident years included in the estimate, the runoff will be more than five years old. For Canadian insurers, for most (but certainly not all) lines of business, the vast majority of claims will have been settled within five years of occurrence. The reserve established after five years will still contain some error. However, since the reserve after five years is generally quite small, the error in estimating the 'true' required reserve should also be small relative to the initial error.

The study is conducted for all lines of business combined since the solvency of the company, the value of the firm, and investor and public confidence are dependent on the overall performance of the company. No adjustment is made for discounting since reserves for the period 1975-1986 were established on a basis which ignored discounting.

In an environment in which interest earned on reserves is accounted for, actual loss reserves could be smaller. Similarly, to the extent that there is an implicit offset of interest and future inflation, any inflationary increases in subsequent payments make reserves appear deficient.

The number of companies in each category is given below:

$\begin{array}{lrrr} & \text { Domestic } & \text { Foreign } & \text { Total } \\ \text { Large Insurers } & 24 & 25 & 49 \\ \text { Medium Insurers } & 23 & 24 & 47 \\ \text { Small Insurers } & 23 & 21 & 44 \\ \text { Reinsurers } & 6 & 23 & 29 \\ \text { Total } & & & \\ & 76 & 93 & 169\end{array}$

\section{The Model}

Let $E_{i}$ denote the estimate in year $i$ of outstanding losses in respect of all accident years $i$ and prior. Let $U_{i}$ denote the estimate made in year $i+5$ of outstanding losses at the end of year $i$ for accident years $i$ and prior. In the analysis in this paper, $U_{i}$ is treated as the 'true' level of outstanding losses at the end of accident year $i$ and $E_{i}$ is an estimate of this true 
value. Of course $U_{i}$ is itself an estimate; but, at year $i+5$, all accident year values are at least 5 years mature. For lines of business that are not too long-tailed, the estimate at year $i+5$ will be reasonably accurate, or at least significantly more accurate than the estimate $E_{i}$ made at year $i$.

The excess/deficiency of the extimate $E_{i}$ is defined as $\left(E_{i}-U_{i}\right) / U_{i}$. It is measured as a fraction of the 'true' value. For positive values of $E_{i}$ and $U_{i}$, the excess/deficiency only takes on values greater than -1 . For the purpose of the statistical analysis described below we transform the excess/deficiency to obtain values taking on all possible values of the real line.

Let $X_{i}=100 \log \left(E_{i} / U_{i}\right)$. Then $E_{i}=U_{i} e^{X_{i} / 100}$, resulting in a simple multiplicative model for the estimate $E_{i}$. Explanatory variables are now introduced and a statistical analysis of the values of $X i$ for all 12 years and for all 169 companies in the data is carried out. The explanatory (categorical) variables in the analysis are:

\begin{tabular}{|c|c|c|c|c|c|c|}
\hline Year: & $y_{i}$, & $i$ & $=$ & $1975,1976, \ldots, 1986$ & 12 & 169 \\
\hline Size: & $s_{j}$ & $j$ & $=$ & Small, Medium, Large, Reinsurer & 4 & \\
\hline Type: & $t_{k}$ & $k$ & $=$ & Domestic, Foreign & 2 & \\
\hline Company: & $c_{i}$ & $l$ & $=$ & company identifiers & & \\
\hline
\end{tabular}

Using a standard analysis of variance (ANOVA) procedure we examine the model

$$
X_{i j k l}=\stackrel{1}{\mu}+y_{i}+s_{j}+t_{k}+\alpha_{i j}+\alpha_{i k}+\alpha_{j k}+\alpha_{i j k}+\epsilon_{i j k l}
$$

where $\mu$ is the overall mean level of $X_{i j k l}, y_{i}$ is the effect of year $i, s_{j}$ is the effect of size $j, t_{k}$ is the effect of type $k$. The quantities $\alpha_{i j}, \alpha_{i k}, \alpha_{j k}$ and $a_{i j k}$ represent the interaction terms of year, type and size. Finally $\epsilon_{i j k i}$ represents the residual 'error' and has mean 0 and variance $\sigma^{2}$. It represents that part of $X_{i j k l}$ that cannot be explained by the above mentioned factors and their interactions.

The results of the analysis of variance are shown in Table 1. It shows that, at a $5 \%$ significance level: 
i) each of year and size are statistically significant explanatory variables;

ii) type is not significant,

iii) only one two-factor interaction is significant, namely type and size; and

iv) the three-way interaction is not significant.

Nonsignificance of some of the interaction terms means that the factor 'year' is independent of size and type. However, the interaction between size and type is significant.

Using the reduced model

$$
X_{i j k l}=\mu+y_{i}+s_{j}+t_{k}+\alpha_{j k}+\epsilon_{i j k l}
$$

results in the analysis of variance table given in Table 2 . From Table 2, it can be seen that the $R^{2}=10.7 \%$, meaning that only $10.7 \%$ of the total variance can be explained by the effects of year, type and size.

TABLE 1

ANALYSIS OF VARIANCE FOR EXCESS/DEFICIENCY

\begin{tabular}{lrrrrr}
$\begin{array}{l}\text { Source of } \\
\text { Variation }\end{array}$ & $\begin{array}{r}\text { Sum of } \\
\text { Squares }\end{array}$ & $\%$ & d.f. & F-ratio & $\begin{array}{r}\text { Sig. } \\
\text { Level }\end{array}$ \\
\hline Year & 213,846 & 6.0 & 11 & 11.46 & 0.00 \\
Size & 126,675 & 3.6 & 3 & 24.89 & 0.00 \\
Type & 2,886 & 0.1 & 1 & 1.70 & 0.19 \\
& & & & & \\
Year Type & 12,345 & 0.3 & 11 & 0.66 & 0.78 \\
Year Size & 33,728 & 1.0 & 33 & 0.66 & 0.96 \\
Type Size & 34,494 & 1.0 & 3 & 6.78 & 0.00 \\
Year Type Size & 31,698 & 0.9 & 33 & 0.57 & 0.98 \\
Model & 455,673 & 12.9 & & & \\
Error & $3,083,677$ & 87.1 & & & \\
Total & & & & &
\end{tabular}


TABLE 2

ANALYSIS OF VARIANCE FOR EXCESS/DEFICIENCY

\begin{tabular}{lrrrrr}
$\begin{array}{l}\text { Source of } \\
\text { Variation }\end{array}$ & $\begin{array}{r}\text { Sum of } \\
\text { Squares }\end{array}$ & $\%$ & d.f. & F-ratio & $\begin{array}{r}\text { Sig. } \\
\text { Level }\end{array}$ \\
\hline Year & 213,846 & 6.0 & 11 & 11.65 & 0.000 \\
Size & 126,675 & 3.6 & 3 & 25.31 & 0.00 \\
Type & 2,886 & 0.1 & 1 & 1.73 & 0.19 \\
Type Size & 34,417 & & 3 & 6.88 & 0.00 \\
Model & 377,824 & 10.7 & & & \\
Error & $3,539,351$ & 89.3 & & & \\
Total & $3,539,351$ & 100.0 & & &
\end{tabular}

TABLE 3

ANALYSIS OF VARIANCE FOR EXCESS/DEFICIENCY

\begin{tabular}{lrrrrr}
$\begin{array}{lrrrr}\text { Source of } \\
\text { Variation }\end{array}$ & $\begin{array}{r}\text { Sum of } \\
\text { Squares }\end{array}$ & $\%$ & d.f. & F-ratio & $\begin{array}{r}\text { Sig. } \\
\text { Level }\end{array}$ \\
\hline Year & 213,846 & 6.0 & 11 & 16.00 & 0.000 \\
Size & 126,675 & 3.6 & 3 & 34.76 & 0.00 \\
Type & 2,886 & 4.6 & 1 & 2.38 & 0.19 \\
Type Size & 34,417 & 1.0 & 3 & 9.44 & 0.00 \\
Company & $1,055,063$ & 29.8 & 161 & 5.39 & 0.00 \\
Model & $1,432,888$ & 40.5 & & & \\
Error & $2,106,463$ & 59.5 & & & \\
Total & $3,539,351$ & 100.0 & & &
\end{tabular}

Table 2 indicates that although the variables 'year', 'type' and 'size' play a significant role in explaining the variation of loss reserves, they collectively explain only $10.7 \%$ of the total observed variability.

In order to test the hypothesis that individual companies consistently over- or under- 
reserve, the variable 'company' was introduced as an additional explanatory variable. The result are given in Table 3 .

Table 3 indicates that an additional $29.8 \%$ of the total variability can be explained by the variable 'company'. This suggests that individual companies are consistently over- or under-reserved (after account is taken of type and size) year after year. Although some consistent over-or under-reserving should be expected since the reserves in successive years are correlated, the data indicate the degree of consistency is high.

The model in Table 3 explains $40.5 \%$ of the variation leaving $59.5 \%$ unexplained. This unexplained variation is due to stochastic error and possible non-stochastic error for reasons that are not (but could possibly be) incorporated into a model.

\section{The Results}

The least squares estimates for 'year', 'size' and 'type' in the model used in Table 3 are given in Table 4. Because of the significant interaction between 'size' and 'type', the (apparently nonsignificant) main effect 'type' remains in the model.

Table 4 indicates that the average level of reserves established during the period 19751985 was almost $1 \%(\exp (0.0093)-1)$ in excess in the level required. It also indicates that there was a dramatic cyclical effect on reserve levels.

Strazewski (1984) reports that the Insurance Services Office estimated that propertyliability companies in the United States were under-reserved by $10 \%$. Our analysis, based on data through 1987, indicates that for Canada, reserves in 1982 were $9.3 \%$ (exp $(0.0093$ 0.1067)-1) deficient.

Although there was no apparent difference between domestic and foreign insurers overall, statistically significant interactions between 'type' and 'size' arose as a result of the large variations shown. 
TABLE 4

RESULTS OF ANALYSIS OF VARIANCE Means of Main Effect:

\begin{tabular}{|c|c|c|}
\hline Overall & $\begin{array}{c}\text { No. of } \\
\text { Observations }\end{array}$ & $\begin{array}{r}\text { Overall } \\
\text { Mean }\end{array}$ \\
\hline & 1914 & $0.83 \%$ \\
\hline $\begin{array}{l}\text { Year of } \\
\text { Reserve }\end{array}$ & $\begin{array}{c}\text { No. of } \\
\text { Observations }\end{array}$ & $\begin{array}{l}\text { Mean } \\
\text { Effect }\end{array}$ \\
\hline 1975 & 150 & $-6.58 \%$ \\
\hline 1976 & 145 & $6.19 \%$ \\
\hline 1977 & 149 & $19.27 \%$ \\
\hline 1978 & 151 & $22.63 \%$ \\
\hline 1979 & 156 & $11.88 \%$ \\
\hline 1980 & 163 & $-1.02 \%$ \\
\hline 1981 & 165 & $-5.67 \%$ \\
\hline 1982 & 167 & $-10.67 \%$ \\
\hline 1983 & 166 & $-3.73 \%$ \\
\hline 1984 & 167 & $-7.58 \%$ \\
\hline 1985 & 166 & $-8.64 \%$ \\
\hline 1986 & 169 & $-0.53 \%$ \\
\hline \multicolumn{3}{|l|}{$\begin{array}{l}\text { Type of } \\
\text { Company }\end{array}$} \\
\hline Dontestic & 835 & $0.36 \%$ \\
\hline Foreign & 1079 & $1.35 \%$ \\
\hline \multicolumn{3}{|l|}{$\begin{array}{l}\text { Sixe of } \\
\text { Company }\end{array}$} \\
\hline Small & 486 & $13.13 \%$ \\
\hline Medium & 543 & $0.50 \%$ \\
\hline Large & 577 & $-1.97 \%$ \\
\hline heinsurer & 308 & $-12.20 \%$ \\
\hline
\end{tabular}

\begin{tabular}{llcr}
\multicolumn{4}{c}{ Means of Interaction Terms } \\
Type & Size & No, of Observations & Mean \\
Domestic & Small & 218 & $3.88 \%$ \\
& Medium & 265 & $-0.15 \%$ \\
& Large & 284 & $1.66 \%$ \\
& Reinsurer & 68 & $-14.39 \%$ \\
Foreign & & & \\
& Small & 268 & $20.65 \%$ \\
& Medium & 278 & $1.13 \%$ \\
& Large & 293 & $-5.49 \%$ \\
& Reinsurer & 240 & $-11.58 \%$
\end{tabular}


We have not shown the 169 individual company effects after accounting for the group effects. Listing the companies and their individual effects serves no useful purpose for us. However, knowledge of the individual effects can be very useful to individual companies' managements and loss reserve specialists and others, as discussed below.

\section{Conclusions}

The results of this study show that a significant amount (40.5\%) of the variability can be easily explained. First, reserve levels for property-casualty companies follow a cyclical pattern. Furthermore there are general differences in reserve levels for companies of different sizes and between direct insurers and reinsurers.

Almost three-quarters of the explained variation comes from the individual companies, irrespective of type, size, or year of valuation. The most important observation is that the individual loss reserve specialist in a given company has consistently over-or under-reserved.

This suggests that any efforts by managements, professions or regulatory authorities should be aimed at the individual company level. Consistent under- or over-reserving may be a result of intentionally trying to improve the apparent financial situation of the company. Greater independence of the loss reserve specialist may provide more objective estimates. The 'appointed actuary' position created through the new act governing insurers and other financial institutions in Canada may improve the situation.

Similarly a tendency for reserve excesses and deficiencies to follow a cyclical pattern suggests that insurers strengthen reserves when they can afford it. This is inconsistent with an objective assessment of loss reserves. Again, independence of the loss reserve specialist may help this situation. Finally, methods of loss reserves that are linked to loss ratios would appear to be inappropriate since premiums are subject to cyclical behaviour as a result of competitive pressure. 


\section{References}

Aiuppa, T. A., and Trieschmann, J. S. (1987) An Empirical Analysis of the Magnitude and Accuracy of Incurred-But-Not Reported Reserves, The Journal of Risk and Insurance, 54, 100-118.

Anderson, D. R. (1971) Effects of Under and Overvaluation in Loss Reserves, The Journal of Risk and Insurance, $98,585-600$.

Ansley, C. F. (1978) Automobile Liability Insurance Reserve Adequacy and the Effect on Inflation, CPCU Journal, 91, 105-112.

Balcarek, R. J. (1975) Loss Reserve Deficiencies and Underwriting Results, Best's Review (Property/Casualty Edition) 76, 21-22, 88.

Conference of Consulting Actuaries (1992), Report of the Task Force on Insurance Solvency in the United States of America, The Casualty Actuary, 3, Oct. 1992.

de Jong, P. and Zehnwirth, B. (1983) Claims Reserving, State-space Models and the Kaiman Filter, Journal of the Institute of Actuaries, 110,157-181.

Forbes, S. W. (1969) Automobile Bodily Injury Liability Loss Reserving Techniques and Simulation, Journal of Risk and Insurance, 36, 597-614.

Forbes, S. W. (1970) Loss Reserving Performance within the Regulatory Framework, The Journal of Risk and Insurance, 37, 527-538.

Grace, E. V. (1990) Property-Liability Insurer Reserve Errors: A Theoretical and Empirical Analysis, Journal of Risk and Insurance, 57, 28-46.

Hafling, D. H. (1981) Incurred but Note Reported Losses, CPCU Journal, 34, 54-57.

Lamm-Tennant, J., Starks, L. T., Stokes, L. (1992) An Empirical Bayes Approach to Estimating Loss Ratios, Journal of Risk and Insurance, 59, 426-442.

Loomis, C. J. (1984) The Earnings Magic at American Express, Fortune, June 25, 58-61.

Panjer, H. H. and Brown, R. L. (1992) An Analysis of Loss Reserves in Canada, Transactions of the 2fth International Congress of Actuaries, 2, 1-13.

Pentikäinen, T., Rantala, J. (1992) A Simulation Procedure for Comparing Different Claims Reserving Methods, ASTIN Bulletin, 22, 191-216.

Renshaw, A.E. (1989) Chain ladder interactive modelling (Claims reserving and GLIM). Journal of the Institute of Actuaries, 116, 559-587. 
Smith, B. (1980) An Analysis of Auto Liability Loss Reserves and Underwriting Results, The Journal of Risk and Insurance, 47 305-320.

Stanard, J.N. (1985) A simulation test of prediction errors of loss reserve estimation techniques. Proceedings of the Casualty Actuarial Society, 72, 124-148.

Strazewski, L. (1984) Reserve Deficiencies Actual: ISO, Business Insurance January 16, 2.

Taylor, C.G. (1986) Claim reserving in non-life insurance. North-Holland, Amsterdam.

van Eeghen, J. (1981) Loss reserving methods. Surveys of actuarial studies 1, NationaleNederlanden N.V., Rotterdam.

Verrall, R. J. (1989a) Bayesian Linear Models and the Claims Run-Off Triangle, Actuarial Research Paper No. 7, Department of Actuarial Science and Statistics, City University London.

Verrall, R.J. (1989b) State space representation of the Chain Ladder linear model. Journal of the Institute of Actuaries, 116, 589-609.

Verrall, R.J. (1990) Bayes and empirical Bayes estimation for the Chain Ladder model. ASTIN Bulletin.

Weiss, M. (1985) A Multivariate Analysis of Loss Reserving Estimates in Property-Liability Insurance Companies, Journal of Risk and Insurance, 52, 199-221.

Zehnwirth, B. (1985) Interactive Claims Reserving Forecasting System, Insureware, St. Kilda, Australia. 